\title{
Primary chemoradiation with cisplatin versus cetuximab for locally advanced head and neck cancer: a retrospective cohort study
}

\author{
Il Seok Daniel Jeong ${ }^{1}$, Huan Mo ${ }^{2}$, Anthony Nguyen ${ }^{3}$, Esther G. Chong ${ }^{4}$, Hsin Hsiang Clarence Tsai ${ }^{1}$, \\ Justin Moyers ${ }^{3}$, Matthew Kim ${ }^{4}$, Curtis Lacy ${ }^{3}$, Vivek Shah ${ }^{4}$, Eric Lau ${ }^{3}$, Yi Xu $^{5}$ and Huynh Cao $^{3^{*}}$
}

\begin{abstract}
Objective: To explore the efficacy of primary chemoradiation with cisplatin versus cetuximab with respect to HPV/ p16 and smoking statuses.

Methods: We retrospectively reviewed patients from our center with locally advanced non-nasopharyngeal head and neck squamous cell carcinoma (HNSCC) who received primary chemoradiation with cisplatin or cetuximab between 2006 and 2018.

Results: The median OS for cisplatin $(n=66)$ was not reached versus 132 months when treated with cetuximab $(n=55)(p=0.03)$. For HPV/p16-positive patients, we found the median OS for cisplatin $(n=34)$ was not reached versus 60 months with cetuximab $(n=21)(p=0.036)$. In the smoking group, the median OS was not reached in the cisplatin group $(n=44)$ versus 60 months when treated with cetuximab $(n=32)(p=0.03)$.
\end{abstract}

Conclusion: HPV/p16-positive and smoking cohorts treated with cisplatin-based chemoradiotherapy had a significantly better OS versus cetuximab.

Keywords: Head and neck squamous cell carcinoma, Cisplatin, Cetuximab, Chemoradiation therapy, HPV/p16

\section{Background}

Head and neck squamous cell carcinoma (HNSCC) is the sixth most common cancer in the world, with 650,000 new cases diagnosed each year. Tobacco use, heavy alcohol intake, and carcinogenic human papillomavirus (HPV) are strong risk factors [1, 2]. HPV-positive HNSCC responds favorably to chemoradiation leading to improved prognosis [3, 4].

Patients who have locoregionally advanced disease at diagnosis receive combined modality therapy with a platinum-based chemotherapy backbone; $[5,6]$ however,

\footnotetext{
*Correspondence: HCao@llu.edu

${ }^{3}$ Department of Hematology and Oncology, Loma Linda University Medical Center, 11175 Campus Street, CSP 11015, Loma Linda, CA 92354 USA

Full list of author information is available at the end of the article
}

in 2006, cetuximab, a chimeric monoclonal antibody and an epidermal growth factor receptor (EGFR) inhibitor, was approved with radiation therapy for locoregionally advanced HNSCC. The addition of cetuximab to radiotherapy improved survival in HNSCC [7]. Cetuximab has been proposed as an alternative to platinum chemotherapy due to a more favorable toxicity profile and improved tolerability in those with renal disease or in the elderly. However, two recent multi-centers randomized clinical trials in patients with HPV associated HNSCC have demonstrated both inferior overall survival of patients who received cetuximab based instead of cisplatin-based radiotherapy and no difference in terms of toxicity profile $[8,9]$. One hypothesis for the decreased efficacy of cetuximab in HPV associated HNSCC is the decreased dependence on the EGFR pathway compared to HPVnegative tumors [10].

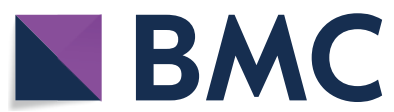

(c) The Author(s) 2020. This article is licensed under a Creative Commons Attribution 4.0 International License, which permits use, sharing, adaptation, distribution and reproduction in any medium or format, as long as you give appropriate credit to the original author(s) and the source, provide a link to the Creative Commons licence, and indicate if changes were made. The images or other third party material in this article are included in the article's Creative Commons licence, unless indicated otherwise in a credit line to the material. If material is not included in the article's Creative Commons licence and your intended use is not permitted by statutory regulation or exceeds the permitted use, you will need to obtain permission directly from the copyright holder. To view a copy of this licence, visit http://creativeco mmons.org/licenses/by/4.0/. The Creative Commons Public Domain Dedication waiver (http://creativecommons.org/publicdomain/ zero/1.0/) applies to the data made available in this article, unless otherwise stated in a credit line to the data. 
We aimed to compare real-world outcomes of cisplatin-based versus cetuximab-based primary chemoradiation in an academic setting at Loma Linda University Medical Center. We also re-analyzed the HNSC dataset from The Cancer Genome Atlas (TCGA) [11] to clarify the relationship between HPV and EGFR statuses in HNSCC.

\section{Materials and methods}

\section{Data collection}

ICD-9, ICD-10, and CPT codes were used to search the Loma Linda University Health Cancer Registry to find patients diagnosed with HNSCC between 2006 and 2018. This study was approved by Loma Linda University Medical Center Institutional Review Board (IRB Approval \#: 5180298). The following data were obtained for each patient: age, sex, smoking status, primary malignancy site, pathology report, staging, type of radiation and number of radiation treatments, chemotherapy regimen, and number of chemotherapy cycles completed. Radiation dose ranged between 48 and $72 \mathrm{~Gy}$.

\section{Patient selection}

Eligible patients were between the ages of 18-99 years who had locally advanced head and neck cancer with initial stages III-IVB receiving concurrent chemoradiation with either cisplatin or cetuximab as first-line therapy. Primary tumors of the oral cavity, oropharynx, and hypopharynx/larynx were included. We excluded those who underwent surgery as first-line therapy, concurrent chemoradiation as second-line or salvage therapy for recurrent or metastatic disease, underlying malignancy other than head and neck cancer, those who did not receive either cisplatin or cetuximab as part of chemotherapy regimen, and nasopharyngeal tumors.

Cases that met inclusion criteria were then separated by chemotherapy received. Categorical variables analyzed included smoking and HPV/p16 statuses, which was abstracted from pathology reports consistent with College of American Pathologists recommendation [12]. p16 ${ }^{\mathrm{INK} 4 \mathrm{a}}$ immunohistochemistry stain and HPV in situ hybridization were performed in Clinical Laboratory Improvement Amendments regulated laboratories with in vitro diagnostic antibodies. Patients with a smoking history were further categorized into light smoking ( $<10$ pack-years) and heavy smoking ( $\geq 10$ pack-years). Comorbidities was considered positive if patients had $\geq 2$ comorbidities and negative if $<2$.

\section{Endpoints and statistics}

Primary endpoint was overall survival (OS) by treatment with either cisplatin or cetuximab. OS was analyzed by the Kaplan-Meier and log-rank methods. Survival was compared by univariate Cox proportional hazards model between treatment groups. Survival analyses were stratified by HPV/p16 and smoking statuses. Statistical analysis with $\mathrm{p}$-values of $<0.05$ was considered significant. Statistics were performed with R packages "survival" and "survminer" (Vienna, Austria).

\section{TCGA data and analysis}

Publicly available data for clinical and phenotypic information, gene expression (by RNA sequencing), and copy number (generated by the Affymetrix Genome-Wide Human SNP Array 6.0 platform) data of the HNSC cohort from TCGA were obtained from University of California Santa Cruz Xena Browser. Gene expression data were represented as RSEM scores, which were $\log 2-$ transformed normalized counts [13]. The copy number estimated values are thresholded to $-2,-1,0,1,2$, representing homozygous deletion, single copy deletion, diploid normal copy, low-level copy number amplification, or high-level copy number amplification reported by GISTIC2 [14, 15]. EGFR copy number statuses were grouped to amplified (GISTIC2 score $\geq 1$ ) and nonamplified (GISTIC2 score $\leq 0$ ). HPV status was obtained from the supplementary information of the original Nature article [11], and most of them were ascertained by the presence of HPV transcripts or genomes by whole genome/exome and RNA sequencing. All analyses were performed on KNIME Analytics.

\section{Results}

\section{Patient and tumor characteristics}

A total of 1545 patients were retrieved from the LLUMC Cancer Registry between 2006 and 2018. Of these patients, 121 met inclusion criteria and were further divided into cisplatin $(n=66)$ and cetuximab $(n=55)$ cohorts (Fig. 1). A summary of demographic information can be found in Table 1 .

\section{Cisplatin chemoradiation improves OS}

The median OS for patients receiving cisplatin was not reached compared to 132 months in the cetuximab group $(\mathrm{p}=0.03$, Hazard Ratio $[\mathrm{HR}]=0.44,[95 \%$ confidence interval (CI), 0.20-0.95]) (Fig. 2a).

\section{Improved OS in HPV/p16-positive cancer treated with cisplatin-based chemoradiation}

Among the patients who were HPV/p16-positive $(n=55)$, those who received cisplatin chemoradiation had longer OS. The median OS for patients with HPV/ p16-positive cancer treated with cisplatin-based therapy was not reached compared to 60 months for HPV/ p16-positive cancer treated with cetuximab $(\mathrm{p}=0.036$, $\mathrm{HR}=0.25$ [95\% CI 0.061-1.00]) (Fig. 2b). 


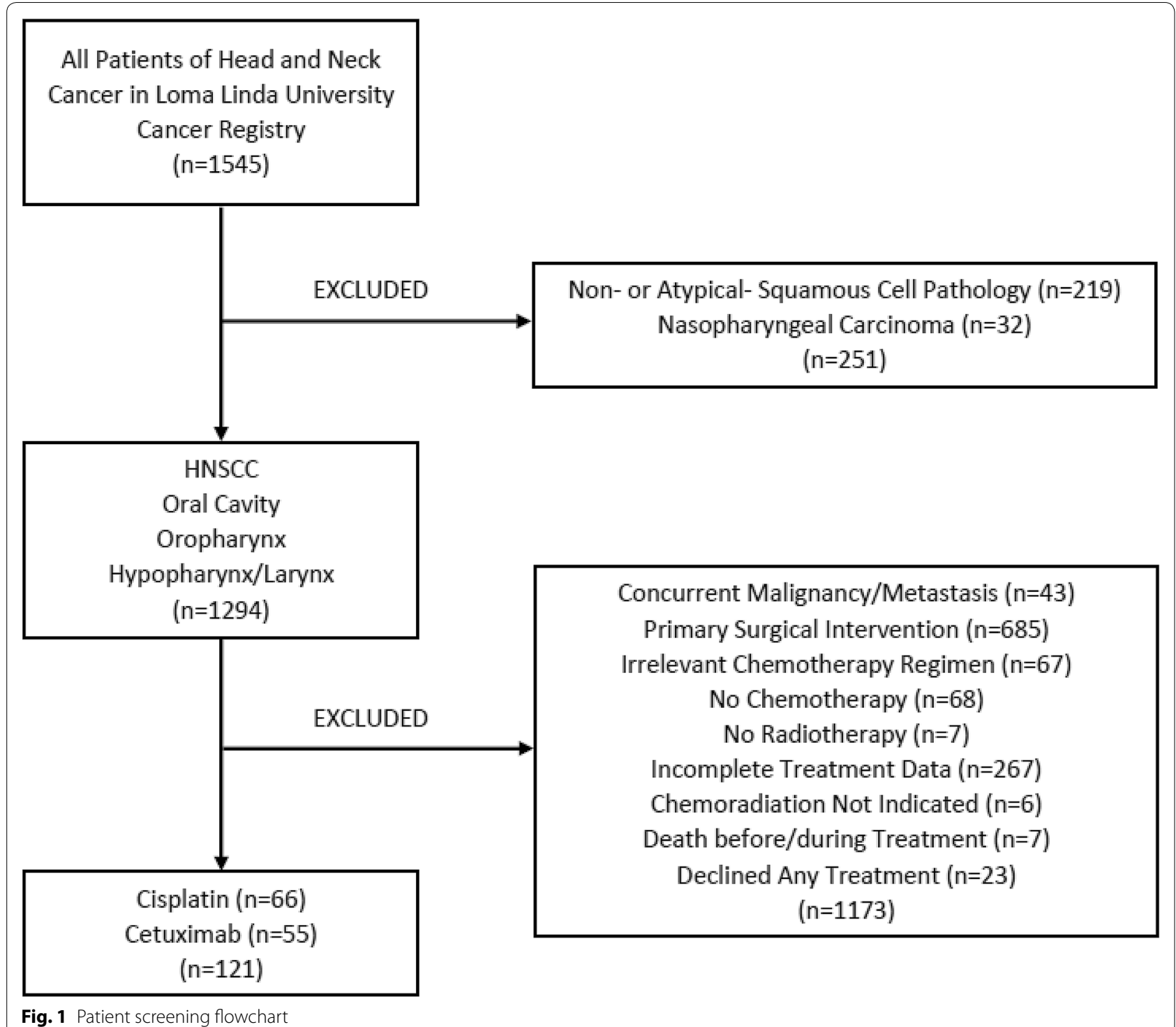

\section{Cisplatin chemoradiation improved OS in patients with positive smoking history}

In patients with a smoking history $(\mathrm{n}=76)$, cisplatinbased radiotherapy was associated with longer OS. The median OS in the cisplatin group was not reached, while that in the cetuximab cohort was 60 months $(\mathrm{p}=0.03, \mathrm{HR}=0.36$ [95\% CI 0.14-0.93]) (Fig. 2c).

\section{Age and outcomes}

Within our study population, patients in the cisplatin group were significantly younger than those in the cetuximab group (56.80 versus 60.04, respectively, $p=0.0003)$. Of patients over 60 years old $(n=51)$, those treated with cisplatin had improved OS $(\mathrm{p}=0.035$, $\mathrm{HR}=0.23$, [95\% CI 0.051-1.03]) (Fig. 2d).

\section{TCGA HNSC data re-analysis}

Within TCGA who had HPV-positive cancers, none of the oropharyngeal $(n=22)$ and only one of the overall head and neck patients $(\mathrm{n}=36)$ had the amplified EGFR gene region, whereas within the HPV-negative cohort, six oropharyngeal $(n=11,54.5 \%)$ and 124 of the overall head and neck patients $(n=243,51.0 \%)$ had the amplified $E G F R$ gene region. Fisher's exact tests indicated that the HPV-negative cohort is more likely to possess EGFR amplification in head and neck patients $(\mathrm{p}<0.0001)$. At the transcription level, RNA expressions of the EGFR 
Table 1 Patient characteristics based on primary chemotherapy received

\begin{tabular}{|c|c|c|}
\hline Parameters & Cisplatin $(n=66)$ & Cetuximab $(n=55)$ \\
\hline \multicolumn{3}{|l|}{ Sex } \\
\hline Male & 51 & 39 \\
\hline Female & 15 & 16 \\
\hline \multicolumn{3}{|l|}{ Age } \\
\hline$>60$ & 18 & 33 \\
\hline$\leq 60$ & 48 & 22 \\
\hline Mean \pm SD (Overall 60.09 \pm 10.28) & $56.80 \pm 9.10$ & $60.04 \pm 10.30$ \\
\hline \multicolumn{3}{|l|}{ HPV/p16 } \\
\hline Positive & 34 & 21 \\
\hline Negative & 9 & 7 \\
\hline Not available & 23 & 27 \\
\hline \multicolumn{3}{|l|}{ Primary } \\
\hline Oropharynx & 55 & 45 \\
\hline Hypopharynx/Larynx & 5 & 7 \\
\hline Oral Cavity & 5 & 2 \\
\hline Other & 1 & 1 \\
\hline \multicolumn{3}{|c|}{ Elevated baseline creatinine (>1.3 mg/dL) } \\
\hline Yes & 14 & 13 \\
\hline No & 43 & 38 \\
\hline Not Available & 9 & 4 \\
\hline \multicolumn{3}{|l|}{ Smoking } \\
\hline Smoker & 44 & 32 \\
\hline Light (<10 Pack-Years) & 10 & 4 \\
\hline Heavy ( $\geq 10$ Pack-Years) & 26 & 25 \\
\hline Unknown Pack-Years & 8 & 3 \\
\hline Non-Smoker & 21 & 21 \\
\hline Not Available & 1 & 2 \\
\hline \multicolumn{3}{|l|}{ Comorbidities } \\
\hline Yes ( $\geq 2$ Conditions) & 48 & 35 \\
\hline No (<2 Conditions) & 16 & 16 \\
\hline Not Available & 2 & 4 \\
\hline \multicolumn{3}{|l|}{ T Staging } \\
\hline $\mathrm{T} 1$ & 6 & 11 \\
\hline $\mathrm{T} 2$ & 26 & 20 \\
\hline $\mathrm{T} 3$ & 20 & 14 \\
\hline $\mathrm{T} 4$ & 12 & 9 \\
\hline Tx & 2 & 1 \\
\hline \multicolumn{3}{|l|}{ N staging } \\
\hline No & 3 & 7 \\
\hline N1 & 8 & 8 \\
\hline N2 & 46 & 36 \\
\hline N3 & 7 & 2 \\
\hline$N x$ & 2 & 2 \\
\hline \multicolumn{3}{|l|}{ M staging } \\
\hline Mo & 60 & 51 \\
\hline M1 & 0 & 0 \\
\hline$M x$ & 6 & 4 \\
\hline
\end{tabular}




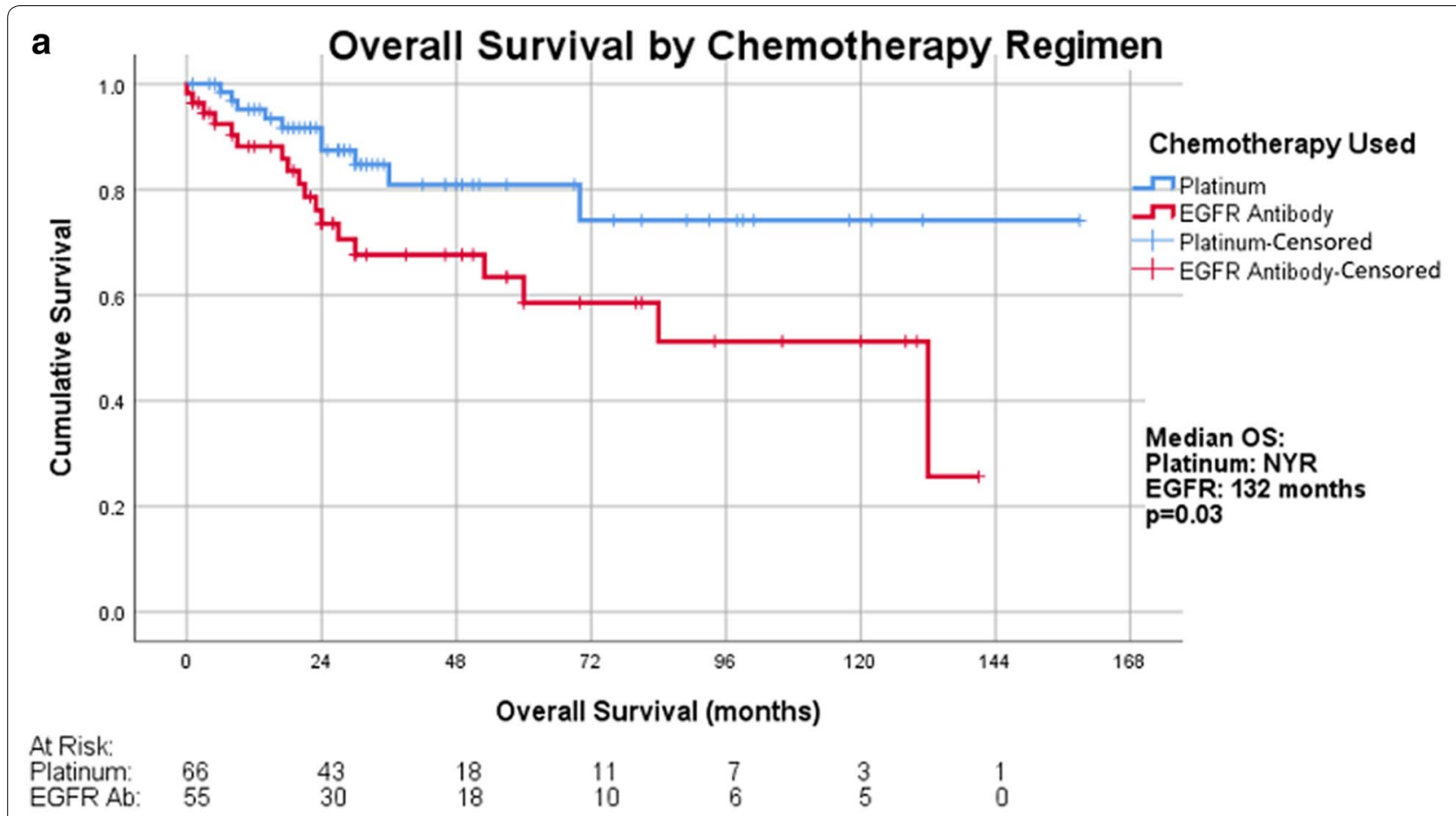

b Overall Survival by Chemotherapy Regimen in HPV-Positive Cases

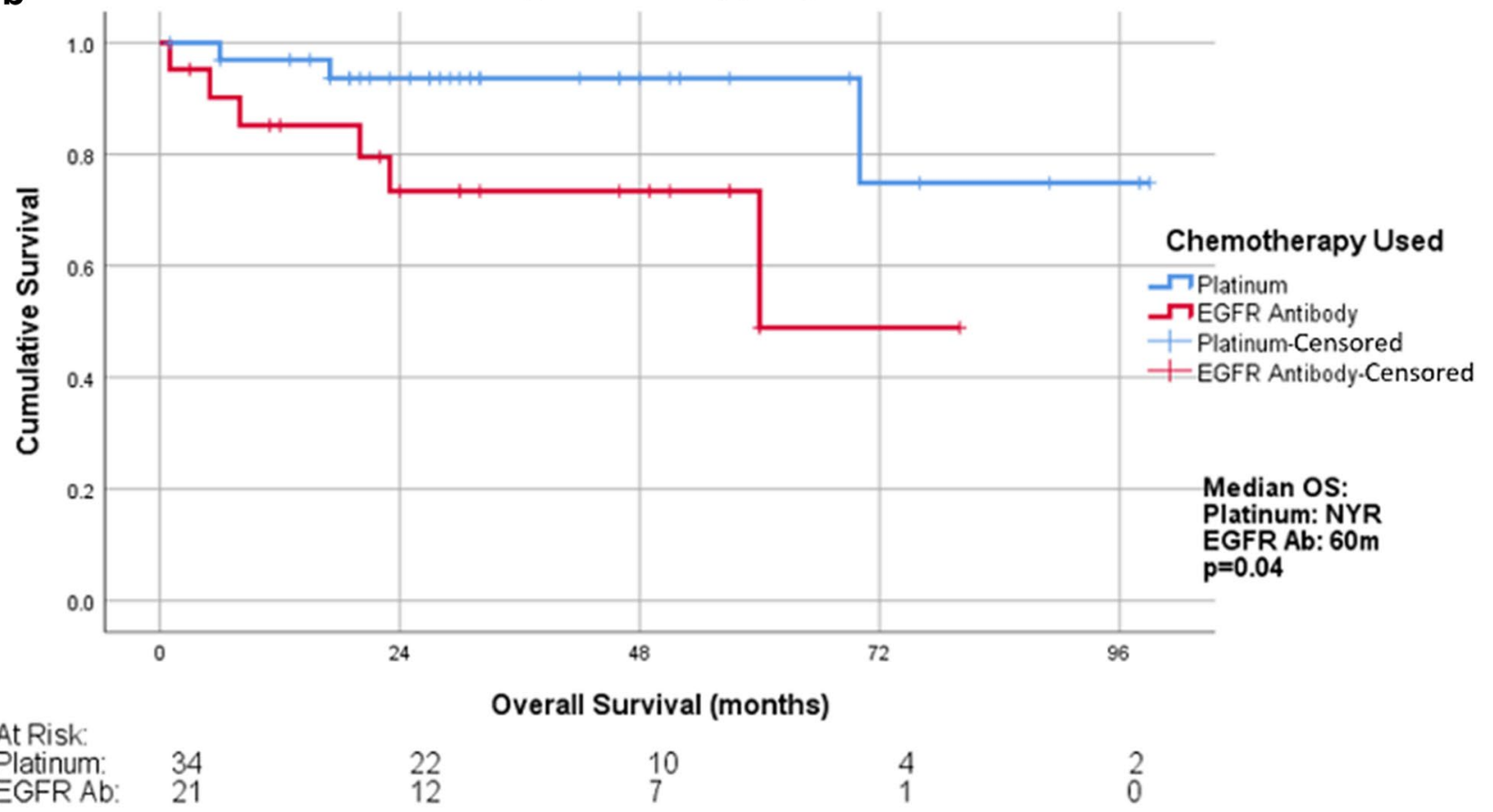

Fig. 2 Overall survival of (a) the overall study population, (b) the HPV/p16-positive population, (c) the smoking population, and (d) the advanced age population that received primary chemoradiation with cisplatin versus cetuximab 


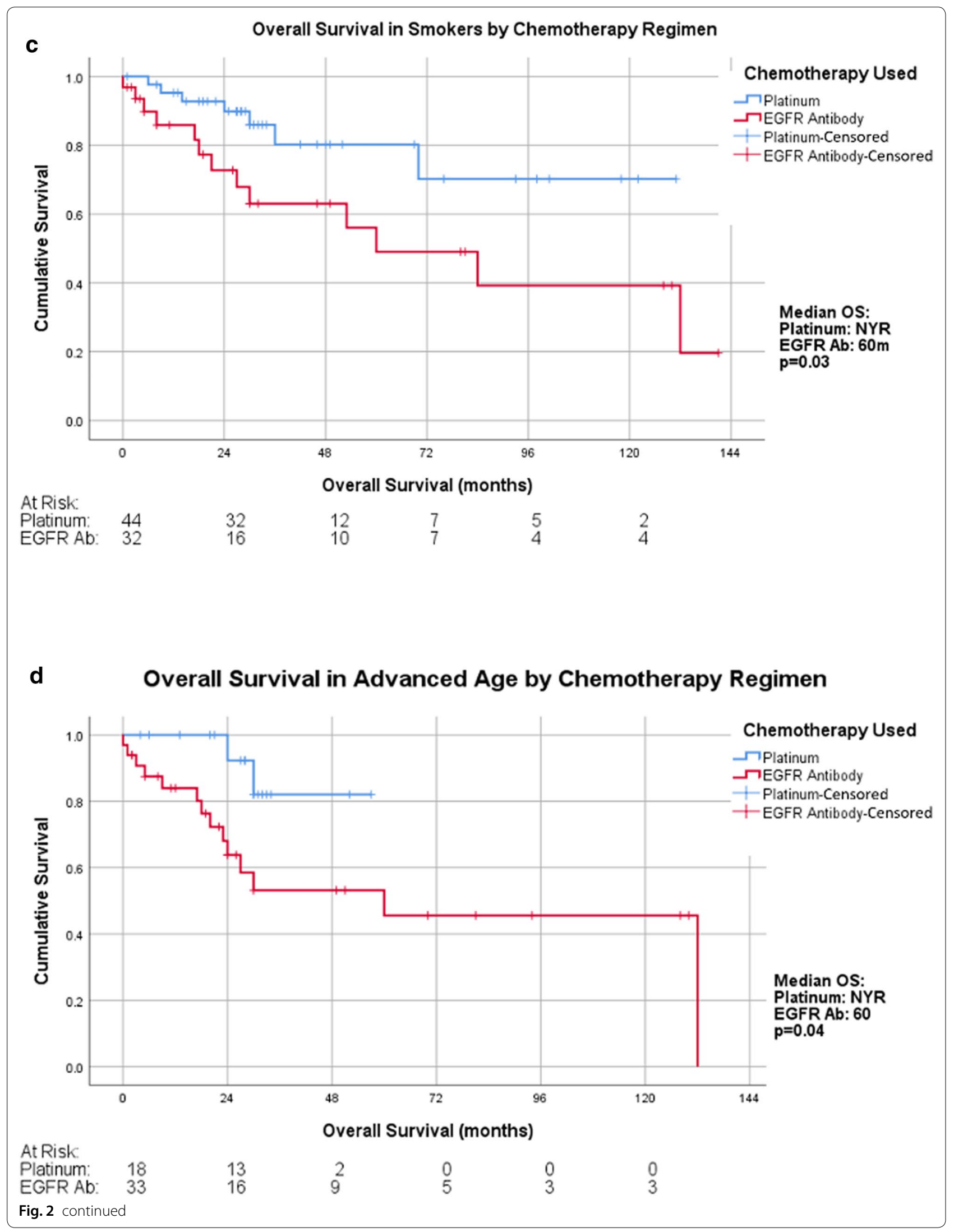




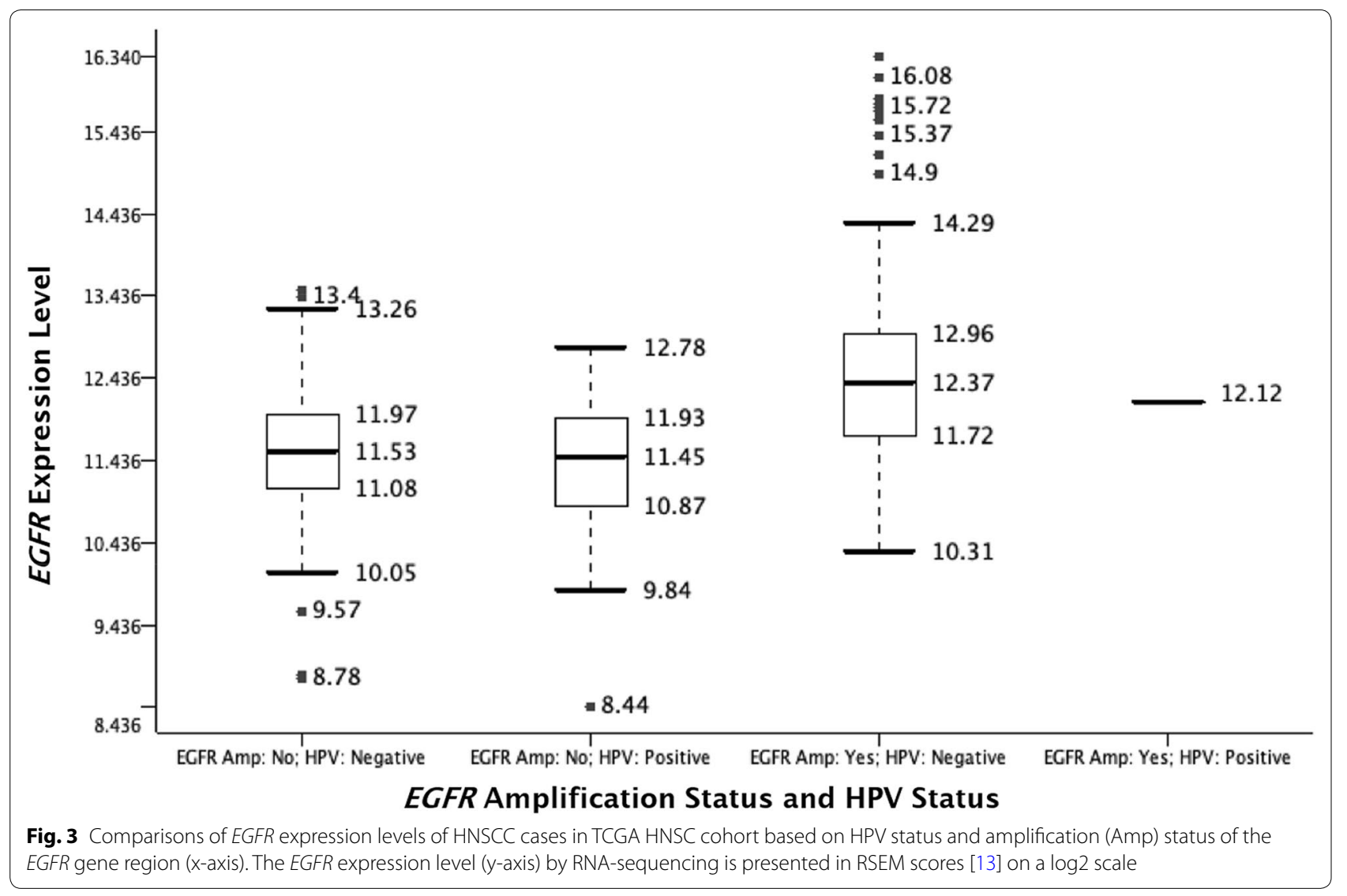

gene in HPV-negative cancers were 1.619 times (95\% CI 1.22 to 2.14) higher than HPV-positive cancers in the overall head and neck cohort ( $\mathrm{p}<0.0001)$ (Fig. 3).

\section{Discussion}

In this single institution retrospective study, we investigated real-world outcomes of locoregionally advanced HNSCC treated with primary chemoradiation with cisplatin versus cetuximab.

Our finding of an OS benefit in HPV/p16-positive cases treated with cisplatin-based therapy compared to cetuximab is consistent with recent phase 3 clinical trial data [ 8 , 9] and national database studies [16-18]. While our study and the VA study by Bauml et al. both found that cetuximab with radiation yields inferior OS, more than $99 \%$ of the VA study population are male and it did not perform a subgroup analysis of HPV status. This growing evidence supports cisplatin-based radiotherapy as the standard of care for patients with HPV/p16-positive cancer with low risk (e.g. non-smokers or $<10$ pack-years smoking history) [9]. We also found the survival benefit with cisplatin therapy to be consistent among those who smoke and those with advanced age. However, cetuximab likely still will play a role in patients with poor renal function, as both even though head to head trials suggested no different overall toxicity, cisplatin consistently demonstrated increased rates of acute kidney injury [8, 9].

Some authors have proposed that the inferior survival outcomes in patients with HPV associated oropharyngeal HNSCC who received concurrent chemoradiation with cetuximab instead of cisplatin might be related to differences in EGFR expression and HPV status. Indeed, we observed that amplification of the EGFR gene region was rare to absent in HPV-positive cancers, while about half of the HPV-negative cases possessed different degrees of amplifications in the TCGA HNSC dataset. Expression of the EGFR gene was also significantly lower in HPV-positive HNSCC, consistent with prior reports [19]. However, EGFR expression has not been shown to be predictive of clinical benefit for anti EGFR therapy in SCCHN, which may be related to the lack of correlation between EGFR expression and autophosphorylation activity [20,21].

The main limitation of this study is that, as a retrospective study, we cannot exclude possible confounding factors that may contribute to inferior OS of cetuximabbased radiotherapy in patients with HPV/p16-positive cancers, such as insufficient renal function that have precluded a cisplatin regimen or the selection bias of 
cetuximab in elderly and frail patients. However, based on our data even the population with advanced age still benefited from high dose cisplatin, and further more patients with at least 2 comorbidities received cisplatin treatment. In conclusion, our result was consistent with the recent prospective randomized clinical trials, which have provided strong evidence of the superiority of cisplatin compared with cetuximab in HPV/p16-positive cancers $[8,9]$.

\section{Abbreviations}

HNSCC: Head and neck squamous cell carcinoma; TCGA: The Cancer Genome Atlas; OS: Overall survival; EGFR: Epidermal growth factor receptor; HPV: Human papillomavirus.

\section{Acknowledgements}

We would like to thank Dr. Bobae Kim and Dr. Lawrence Liu for assisting with manuscript editing.

\begin{abstract}
Authors' contributions
ISDJ collected and processed the data, reviewed literature, wrote and edited the manuscript. HM and JM performed statistical analysis and wrote and edited the manuscript. MK and HCT collected the data and wrote the manuscript. EC, CL, VS, EL and YX helped with literature review, and edited the manuscript. AN and HC designed the study, reviewed literature, and wrote and edited the manuscript. All authors read and approved the final manuscript.
\end{abstract}

\section{Funding}

Not applicable

\section{Availability of data and materials}

The single institute data from this study is available from the corresponding author upon reasonable request.

\section{Ethics approval and consent to participate}

This study was approved by Loma Linda University Medical Center Institutional Review Board (IRB Approval \#: 5180298).

\section{Consent for publication}

Not applicable.

\section{Competing interests}

The authors declare that they have no competing interests.

\section{Author details}

${ }^{1}$ School of Medicine, Loma Linda University, Loma Linda, CA, USA. ${ }^{2}$ Department of Pathology, Loma Linda University Medical Center, Loma Linda, CA, USA. ${ }^{3}$ Department of Hematology and Oncology, Loma Linda University Medical Center, 11175 Campus Street, CSP 11015, Loma Linda, CA 92354, USA. ${ }^{4}$ Department of Medicine, Loma Linda University Medical Center, Loma Linda, CA, USA. ${ }^{5}$ Department of Regenerative Medicine, Loma Linda University Medical Center, Loma Linda, CA, USA.

Received: 13 May 2020 Accepted: 29 July 2020

Published online: 05 August 2020
2. Vokes EE, et al. Head and neck cancer. N Engl J Med. 1993;328(3):184-94.

3. Vigneswaran N, Williams MD. Epidemiologic trends in head and neck cancer and aids in diagnosis. Oral Maxillofacial Surg Clinics North Am. 2014;26(2):123-41.

4. Marur S, et al. HPV-associated head and neck cancer: a virus-related cancer epidemic. Lancet Oncol. 2010;11(8):781-9.

5. Bachaud JM, et al. Combined postoperative radiotherapy and Weekly Cisplatin infusion for locally advanced head and neck carcinoma: Final report of a randomized trial. Int J Radiation Oncol Biol Phy. 1996;36(5):999-1004.

6. Szturz P, Vermorken JB. Treatment of elderly patients with squamous cell carcinoma of the head and neck. Front Oncol. 2016;6:199.

7. Rosenthal DI, et al. Association of human papillomavirus and p16 status with outcomes in the IMCL-9815 phase III registration trial for patients with locoregionally advanced oropharyngeal squamous cell carcinoma of the head and neck treated with radiotherapy with or without cetuximab. J Clin Oncol. 2015;34(12):1300-8.

8. Gillison ML, et al. Radiotherapy plus cetuximab or cisplatin in human papillomavirus-positive oropharyngeal cancer (NRG Oncology RTOG 1016): a randomised, multicentre, non-inferiority trial. Lancet. 2019;393(10166):40-50

9. Mehanna H, et al. Radiotherapy plus cisplatin or cetuximab in low-risk human papillomavirus-positive oropharyngeal cancer (De-ESCALaTE HPV): an open-label randomised controlled phase 3 trial. Lancet. 2019;393(10166):51-60.

10. Oosthuizen JC, Doody J. De-intensified treatment in human papillomavirus-positive oropharyngeal cancer. Lancet. 2019;393(10166):5-7.

11. Lawrence MS, et al. Comprehensive genomic characterization of head and neck squamous cell carcinomas. Nature. 2015;517(7536):576-82.

12. Lewis JS, et al. Human papillomavirus testing in head and neck carcinomas: guideline from the College of American Pathologists. Arch Pathol Lab Med. 2017;142(5):559-97.

13. Li B, Dewey CN. RSEM: accurate transcript quantification from RNA-Seq data with or without a reference genome. BMC Bioinform. 2011;12(1):323.

14. Mermel CH, et al. GISTIC2.0 facilitates sensitive and confident localization of the targets of focal somatic copy-number alteration in human cancers. Genome Biol. 2011;12(4):R41.

15. Zack TI, et al. Pan-cancer patterns of somatic copy number alteration. Nat Genet. 2013;45(10):1134-40.

16. Bauml JM, et al. Cisplatin versus cetuximab with definitive concurrent radiotherapy for head and neck squamous cell carcinoma: an analysis of Veterans Health Affairs data. Cancer. 2019;125(3):406-15.

17. Riaz N, et al. Concurrent chemoradiotherapy with cisplatin versus cetuximab for squamous cell carcinoma of the head and neck. Am J Clin Oncol. 2016;39(1):27.

18. Tang C, et al. Concurrent cetuximab versus platinum-based chemoradiation for the definitive treatment of locoregionally advanced head and neck cancer. Head Neck. 2015;37(3):386-92.

19. Lassen P, Overgaard J, Eriksen JG. Expression of EGFR and HPV-associated p16 in oropharyngeal carcinoma: correlation and influence on prognosis after radiotherapy in the randomized DAHANCA 5 and 7 trials. Radiother Oncol. 2013;108(3):489-94.

20. Kriegs $M$, et al. Analyzing expression and phosphorylation of the EGF receptor in HNSCC. Sci Rep. 2019;9(1):13564.

21. Bossi $P$, et al. Prognostic and predictive value of EGFR in head and neck squamous cell carcinoma. Oncotarget. 2016;7(45):74362-79.

\section{Publisher's Note}

Springer Nature remains neutral with regard to jurisdictional claims in published maps and institutional affiliations.

\section{References}

1. Falk RT, et al. Effect of smoking and alcohol consumption on laryngeal cancer risk in coastal Texas. Cancer Res. 1989:49(14):4024-9. 